initial guidance about the amount of change associated with improvement or worsening, supporting its use in RA care, research and decisionmaking.

Acknowledgements: The CATCH study was designed and implemented by the investigators and financially supported through unrestricted research grants from: Amgen and Pfizer Canada - Founding sponsors since January 2007; AbbVie Corporation and Hoffmann-LaRoche since 2011; Medexus Inc. since 2013; , Merck Canada since 2017, Sandoz Canada, Biopharmaceuticals since 2019,Gilead Sciences Canada since 2020 and Fresenius Kabi Canada Ltd. since 2021. Previously funded by Janssen Biotech from 2011-2016, UCB Canada and Bristol-Myers Squibb Canada from 2011-2018, Sanofi Genzyme from 2016-2017, and Eli Lilly Canada from 2016-2020.

Disclosure of Interests: None declared

DOI: 10.1136/annrheumdis-2021-eular.1663

\section{POS1460-HPR MYRA TOUCH FOR DISEASE ACTIVITY EVALUATION IN RHEUMATOID ARTHRITIS: A COMPARISON OF PATIENT-REPORTED OUTCOMES WITH PHYSICIAN'S ASSESSMENT}

L. H. Eow ${ }^{1}$, J. Yew ${ }^{1}$, K. H. Lee2, S. Selvadurai2, S. M. Liau2, N. Mohd Noor2, A. Mohd2, Y. L. Loh2, S. C. Gun2. ${ }^{1}$ Hospital Tuanku Ja'afar, Medical Department, Seremban, Malaysia; ${ }^{1}$ Hospital Tuanku Ja'afar, Medical Department, Seremban, Malaysia

Background: Patient-reported outcomes (PROs) have become an essential component of patients' assessment in the management of Rheumatoid Arthritis(RA). They have been reported to be at least as informative if not more than physician assessed outcomes.

MyRA Touch was pioneered by the Rheumatology Unit of Hospital Tuanku Jaa'far in Seremban Malaysia in March 2018,to engage and empower all RA patients on their own disease activity monitoring. It is an electronic platform, designed to enhanced the application of electronic patient reported outcomes (ePROs) among RA patients where they examine and record their own painful and/or swollen joints for DAS28 calculation and report their health assessment through Routine Assessment of Patient Index Data with 3 Measures (RAPID 3).MyRA Touch is an applications (App) that is user friendly and available in four major spoken languages (English, Chinese, Malay and Tamil) with an animated version for patients who are illiterate.

Objectives: The objectives of this study are to determine the correlation between:

I)Patient-reported and physician reported DAS28 ESR/CRP

II)RAPID3 and Clinical Disease activity Index (CDAI)

III)RAPID3 and DAS28 ESR/CRP assessed by physician and patient

IV)RAPID3 and inflammatory markers ESR/CRP.

Methods: This was a cross-sectional study carried out in the Rheumatology Unit of Hospital Tuanku Jaa'far. All data entered through MyRA Touch App from April 2018 till April 2020 was analysed.

Results: There were a total of 562 patients who entered the data in the App, $87.9 \%$ were women. The ethnic compositions of the study subjects comprised of Indians (36.7\%) followed by the Malays (34.7\%), Chinese $(26.3 \%)$ and other ethnics $(2.3 \%)$. About half of patients $(59.8 \%)$ were in the $51-70$ age group whereas $22.9 \%, 1.8 \%$ and $15.5 \%$ were in the $31-50,18-30$ and above 70 age groups respectively. The majority of our patients (96\%) were literate. A total of $54.3 \%$ of them received secondary education, $27 \%$ primary, $12.2 \%$ tertiary and $6.6 \%$ did not receive any formal education.

There was a high level of correlation between DAS28 ESR/CRP performed by patient and DAS28 ESR/CRP assessed by physician, ( $r=0.808$ for DAS28 ESR and $r=0.804$ for DAS28 CRP). RAPID3 also showed high level of correlation with CDAl and DAS28 CRP assessed by patient ( $r=0.700$ and $r=0.718$ respectively). There was a moderate correlation between DAS28 ESR/CRP done by physician with RAPID3 ( $r=0.656$ and $r=0.696$ respectively).RAPID3 demonstrated little correlation with inflammatory markers ESR and CRP ( $r=0.141$ and $r=0.171$ respectively).

Conclusion: PROs via DAS 28 (ESR/CRP) and RAPID3 showed moderate to high correlation with disease activity assessed by physician. We can empower patients to perform their own disease assessment by using the MyRA Touch App before seeing their physician and the information provided in the App, can help to reduced consultation time. During the COVID-19 pandemic, telemedicine is very much encouraged. By using the MyRA Touch, patients can assess their own tender and swollen joint count on a homunculus, evaluate their own physical function, health and pain using the RAPID3 parameters. The information obtained from the PROs in the MyRA touch App enables the physician to make a more comprehensive virtual assessment of the patient's condition which helps in treatment decision making. In conclusion, MyRA Touch is an useful tool for disease activity measurement by patient.

\section{REFERENCES:}

[1] Jenny AA, Diana BC, Omar JC, et al. Usefulness of Patients-Reported Outcomes in Rheumatoid Arthritis Focus Group. Hindawi Publishing Corporation Arthritis, vol 2012,Article ID935187.

[2] Ana MO, Clifton OB. Patient Reported Outcomes in Rheumatoid Arthritis Clinical Trials. Curr Rheumato Rep.2015 April;17(4):501.

Disclosure of Interests: None declared

DOI: 10.1136/annrheumdis-2021-eular.1813

\section{POS1461-HPR THE DEVELOPMENT OF A NOVEL EPRO DELIVERY SYSTEM TO MEASURE PATIENT QUALITY OF LIFE IN ROUTINE CLINICAL CARE: AN ANALYSIS OF 5 YEARS OF EXPERIENCE}

$\underline{K}_{\text {K Tymms }}^{1,2}$, T. Smith ${ }^{1}$, C. Deakin ${ }^{1}$, T. Freeman ${ }^{3}$, D. Hoffman ${ }^{3}$, D. Segelov ${ }^{3}$, H. Griffiths ${ }^{1,4}$, S. Ciciriello ${ }^{1,5}$, P. Youssef ${ }^{1,6}$, D. Mathers ${ }^{1,7}$, C. Osullivan ${ }^{1}$, G. Littlejohn ${ }^{1,8} .{ }^{1}$ OPAL Rheumatology, Ltd, Sydney, Australia; ${ }^{2}$ Canberra Rheumatology, Canberra, Australia; ${ }^{3}$ Software 4 Specialists (S4S), Pty Ltd, Sydney, Australia; ${ }^{4}$ Barwon Rheumatology, Services, Geelong, Australia; ${ }^{5}$ Dept. of Rheumatology, Royal Melbourne Hospital, Melbourne, Australia; ${ }^{6}$ Dept. of Rheumatology, Royal Prince Alfred Hospital, Sydney, Australia; ${ }^{7}$ Georgetown Arthritis, Georgetown, Australia; ${ }^{8}$ Dept. of Medicine, Monash University, Clayton, Australia

Background: Registry studies and clinical trials are increasingly incorporating patient reported outcomes (PROs) to measure the full burden of disease and better measure the efficacy and value of medicines; however, the burden of paperbased surveys, time constraints, and privacy concerns impede the widespread use of PROs in routine clinical care.

Objectives: To develop a simple and secure technological solution to incorporate validated $\mathrm{PROs}$ into routine clinical care for patients with rheumatic diseases, and to assess the patient response to functional assessment of chronic illness therapy fatigue (FACIT-F), patient health questionnaire-2 (PHQ-2), and healthcare resource utilization (HCRU) questionnaires delivered using this ePRO method.

Methods: A novel ePRO questionnaire delivery system was developed by Software4Specialists in partnership with OPAL Rheumatology. Validated PRO questionnaires were sent from the patient's electronic medical record (Audit4, Software4Specialists) and delivered to the patient's email address at time intervals specified by the rheumatologist (defaults to quarterly) or completed in the clinic waiting room prior to the consultation using a tablet or the patient's smart phone (in-practice). Completed questionnaires were encrypted and returned directly to the patient's Audit4 electronic medical record held on the clinician's server for review at the next clinical consultation. The link to the PRO questionnaire expired within 28 days if the questionnaire was not completed, and the questionnaires were automatically cancelled if 2 consecutive links expired. This technology was made available to up to 111 rheumatologists located in $42 \mathrm{clin}$ ics in 6 states/territories in Australia, and the use of this technology to furnish the clinical consultation was voluntary for clinicians and patients. Deidentified clinical data was extracted from the servers of participating rheumatologists and aggregated across all sites. ${ }^{1}$ Data collected between April 2016-Dec 2020 was analysed descriptively.

Results: Between April 2016-Dec 2020, 99,505 FACIT-F, PHQ-2 and HCRU questionnaires have been delivered to 5,784 patients from 39 of 42 contributing clinics (93\%). $85 \%$ of questionnaires were delivered via email and $15 \%$ in-practice. Overall, $85 \%$ of patients completed at least one questionnaire, and of all questionnaires sent, $73 \%$ were completed. These rates have remained consistent over time. The completion rates were higher when questionnaires were delivered to patients in-practice compared to email ( $96 \%$ vs $69 \%$ ). Females were more likely to engage with the questionnaires than males $(87 \%$ vs $81 \%$ ), and older patients were slightly more likely to complete all questionnaires delivered. $69 \%$ of questionnaires sent via email were completed on the day they were delivered and $94 \%$ were completed within 7 days. The median (IQR) number of questionnaires completed per patient was $3(1,7)$ and the median (IQR) time since the first questionnaire was completed was 13 months $(5,26)$.

Conclusion: The novel Audit4 ePRO delivery system is an effective tool for incorporating PROs into routine clinical care to capture data directly from the patient on the impact of their condition on their quality of life. The data generated provides a unique opportunity to understand the full burden of disease for patients in the real-world setting and the impact of interventions. 
REFERENCES:

[1] Littlejohn GO, Tymms KE, Smith T, Griffiths HT. Using big data from realworld Australian rheumatology encounters to enhance clinical care and research. Clin Exp Rheum 2020:38(5): 874 -880.

Acknowledgements: The authors acknowledge the members of OPAL Rheumatology Ltd and their patients for providing clinical data for this study, and Software4Specialists Pty Ltd for providing the Audit4 platform.

Disclosure of Interests: Kathleen Tymms: None declared, Tegan Smith: None declared, Claire Deakin: None declared, Tim Freeman: None declared, David Hoffman: None declared, Dana Segelov: None declared, Hedley Griffiths Consultant of: AbbVie, Gilead, Novartis and Lilly., Sabina Ciciriello: None declared, Peter Youssef: None declared, David Mathers: None declared, Catherine OSullivan: None declared, Geoff Littlejohn Consultant of: Over the last 5 years Geoffrey Littlejohn has received educational grants and consulting fees from AbbVie, Bristol Myers Squibb, Eli Lilly, Gilead, Novartis, Pfizer, Janssen, Sandoz, Sanofi and Seqirus

DOI: 10.1136/annrheumdis-2021-eular.2253

\section{POS1462-HPR VALIDITY AND PSYCHOMETRIC CHARACTERISTICS OF THE PSORIATIC ARTHRITIS QUALITY OF LIFE (PSAQOL) QUESTIONNAIRE IN TURKISH POPULATION}

M. T. Duruöz ${ }^{1}$, K. Nas ${ }^{2}$, S. Acer Kasman ${ }^{1}$, E. Uzun ${ }^{3}$, H. H. Gezer ${ }^{1}{ }^{1}$ Marmara University, School of Medicine, Physical Medicine and Rehabilitation Department, Rheumatology Division, Istanbul, Turkey; ${ }^{2}$ Sakarya University School of Medicine, Division of Rheumatology and Immunology, Department of Physical Medicine and Rehabilitation, Sakarya, Turkey; ${ }^{3}$ Sakarya University School of Medicine, Department of Physical Medicine and Rehabilitation, Sakarya, Turkey

Background: Psoriatic Arthritis Quality of Life (PsAQoL) questionnaire was developed to measure quality of life $(\mathrm{QoL})$ in patients with psoriatic arthritis (PsA) (1).

Objectives: The aim of the study was to assess validity and psychometric properties of the PSAQoL questionnaire.

Methods: The translation-back translation method by a bilingual panel was used to translate the original PsAQoL into Turkish (2). Face and content validities were evaluated via cognitive debriefing interviews with 10 PsA patients. Then, patients with PsA according to CASPAR criteria were recruited to assess feasibility, missing data, floor and ceiling effect, reliability, and construct validity. Reliability was assessed by internal consistency. Construct validity was investigated by convergent and divergent validity by assessing the PsAQoL's relations with QoL and non-QoL parameters. For this purpose, demographics, clinical characteristics (shown in Table 1), survey data including PSAQoL, Short form 36, visual analog scales for pain and fatigue, Health Assessment Questionnaire, and Hospital Anxiety and Depression Scale results were recorded.

Results: One hundred and sixty-two patients were recruited and 74 of them had axial PsA. Cognitive debriefing showed the Turkish version of PsAQoL to be clear, understandable, and relevant. It was easy to complete and calculate, with the 3 minutes and 20 seconds, respectively. The Cronbach's alpha coefficient for internal consistency was 0.930 . The questionnaire presented no floor or ceiling

Table 1. Spearman's correlation coefficients of PsAQoL with the other parameters for construct validity

\begin{tabular}{|c|c|c|c|c|c|}
\hline \multicolumn{3}{|l|}{ Convergent } & \multicolumn{3}{|c|}{ Divergent } \\
\hline SF-36 subscales & Rho & Clinical Parameters & Rho & Surveys & Rho \\
\hline Physical component & $-0.744^{\star *}$ & Age & 0.150 & BASDAI (n:74) & $0.355^{\star \star}$ \\
\hline Mental component & $-0.731^{* *}$ & Disease duration & 0.014 & VAS Pain & $0.408^{\star \star}$ \\
\hline Physical functioning & $-0.629^{\star *}$ & Tender joint count & $0.262^{* *}$ & VAS Fatigue & $0.447^{\star \star}$ \\
\hline Physical role limitations & $-0.640^{\star \star}$ & Swollen joint count & $0.170^{\star}$ & $\mathrm{HAQ}$ & $0.533^{\star \star}$ \\
\hline Emotional role limitations & $-0.576^{\star *}$ & MASES & 0.152 & HADS Anxiety & $0.535^{\star \star}$ \\
\hline Vitality & $-0.657^{* *}$ & PASI & -0.032 & HADS Depression & $0.517^{\star \star}$ \\
\hline Emotional well-being & $-0.590^{* *}$ & DAPSA & $0.409^{\star \star}$ & & \\
\hline Social functioning & $-0.591^{\star *}$ & DAS28 & $0.322^{\star *}$ & & \\
\hline Pain & $-0.535^{\star *}$ & BASMI (n: 61) & 0.032 & & \\
\hline General health & $-0.536^{\star *}$ & & & & \\
\hline
\end{tabular}

${ }^{* *} p<0.001,{ }^{*} p:$ 0.001-0.049.PSAQoL: Psoriatic Arthritis Quality of Life, SF-36: Short Form 36, MASES: Maastricht Ankylosing Spondylitis Enthesitis Score, PASI: Psoriasis Area Severity Index, DAPSA: Disease Activity in PSoriatic Arthritis, DAS28: Disease Activity Score 28, BASMI: Bath Ankylosing Spondylitis Metrology Index, BASDAI: Bath Ankylosing Spondylitis Disease Activity Index, VAS: Visual analog scale, HAQ: Health Assessment Questionnaire, HADS: Hospital Anxiety and Depression Scale. effect ( $<15 \%$ for both) and no missing data. PsAQoL showed good correlations with the QoL scales indicating its convergent validity and moderate to non-significant correlations with the non-QoL parameters supporting its divergent validity (Table 1).

Conclusion: The Turkish version of the PsAQoL is a valid and disease-specific instrument with good psychometric characteristics to assess PsA-specific QoL.

\section{REFERENCES:}

[1] McKenna SP, Doward LC, Whalley D, Tennant A, Emery P, Veale DJ. Development of the PSAQoL: a quality of life instrument specific to psoriatic arthritis. Ann Rheum Dis. 2004;63(2):162-169.

[2] Beaton DE, Bombardier C, Guillemin F, Ferraz MB. Guidelines for the process of cross-cultural adaptation of self-report measures. Spine (Phila Pa 1976). 2000;25(24):3186-3191.

Disclosure of Interests: None declared

DOI: 10.1136/annrheumdis-2021-eular.3311

\section{POS1463-HPR IMPACT OF COVID-19 PANDEMIC ON DISEASE ACTIVITY AND EMOTIONAL WELL-BEING AMONG RHEUMATOID ARTHRITIS PATIENTS: A TELEMEDICINE STUDY}

B. Lucchino $^{1}$, C. Iannuccelli ${ }^{1}$, C. Gioia ${ }^{1}$, G. Dolcini ${ }^{1}$, M. DI Franco ${ }^{1} .{ }^{1}$ Sapienza University of Rome, Dipartimento di Scienze Cliniche Internistiche,

Anestesiologiche e Cardiovascolari, Rome, Italy

Background: By the beginning of March 2020, the pandemic of the novel coronavirus disease (COVID-19) imposed severe lockdown measures opposing the viral spread, limiting non-COVID patients' access to hospitals Previous reports suggest that Rheumatoid Arthritis (RA) patients may experience a worsening of self-reported disease activity following natural disasters. Moreover, RA patients are at increased risk of mood disorders, such as depression and anxiety. An increase of depressive symptoms, anxiety and suicidal rate has been recently reported as result of the lockdown in response to COVID-19.

Objectives: Aims of this study were to investigate the impact of the lockdown measures on disease activity and emotional well-being among patients affected by RA, through a telemedicine approach.

Methods: Patients followed in the "Early Arthritis Clinic" of our hospital were invited to participate to an online survey. They were asked also to invite their best friend (BF), matched for age and sex, to participate the survey, as control group. Moreover, clinical records from the same "Early Arthritis Clinic" cohort were used as pre-pandemic group. The online survey included demographic questions and, for RA patients, the evaluation of perceived pain (P-VAS) and disease activity (DA-VAS) on a scale of 1-10 as well as Rheumatoid Arthritis Disease Activity Index-5 (RADAI-5), a validated self-reported disease activity measure. Both RA patients and BF responded also to validated, psychometric scales for stress vulnerability (Stress Vulnerability Scale-SVS), resilience (Resilience Scale-RS), depression (Zung's depression questionnaire-Zung-D) and anxiety (Zung's anxiety questionnaire-Zung-A) evaluation.

Results: The cohort was composed by 76 RA patients who answered the survey, 32 BF and 170 RA patients from historical cohort. There were no differ ences in demographic characteristics, SVS, RS, Zung-D and Zung-A between RA patients and BF. RADAl-5 correlated positively with SVS $(r=0,29 ; p=0,01)$, P-VAS $(r=0,8 ; p<0,0001)$ and DA-VAS $(r=0,8 ; p<0,0001)$ and negatively with $R S$ $(r=-0,23 ; p=0,04)$. Patients with higher disease activity showed higher classes of Zung-D $(p=0,03)$ and SVS $(p=0,006)$, and lower classes of $R S(p=0,001)$. P-VAS was the only significant predictor of $\operatorname{RADAl}-5(\beta=0,83 ; \mathrm{Cl}=0,53-0,76 ; \mathrm{p}<0,001)$, and the concordance between RADAI-5, P-VAS and DA-VAS was high $(K=0,94 ; p<0,001)$. Compared with the historical cohort, RA patients involved in the survey showed higher frequencies of moderate and elevated disease activity classes $(p<0,001$; Figure 1$)$ despite there were no differences in P-VAS and DA-VAS.

Conclusion: These results suggest that lockdown did not affect differently emotional well-being of RA patients compared to healthy subjects. RA patients with elevated disease activity report higher stress and depressive symptoms, and SVS and RS may be useful to identify patients at higher risk. The higher self-reported disease activity measured through RADAI- 5 may be the result of an overestimation of disease severity by patients, which depends mostly from pain. This should be considered in the assessment of disease activity through telemedicine. 